\title{
Caracterización fenotípica y factores de virulencia en cepas de Aeromonas aisladas de pacientes con enfermedad diarreica aguda en Cuba
}

\author{
Laura Bravo, Anabel Fernández, Judith Ledo, Margarita Ramírez, Adalberto Aguila, \\ Fidel A. Núñez, Luis E. Cabrera y Yanaika Cruz.
}

\section{Phenotypic characteristics and virulence factors in Aeromonas strains isolated from patients with diarrheic disease in Cuba}

Fifty four strains of Aeromonas spp were isolated from patients with acute diarrheic episodes by using Aerokey II and Aeroesquema methods. In vitro antimicrobial susceptibility and virulence factors were analyzed. The most frequently isolated specie was Aeromonas caviae. Over $75 \%$ of strains exhibited resistance to penicillins and cephalosporins; for the other antibiotic groups resistance was under $20 \%$. Twenty six strains (48.1\%) were multiresistant. At least one virulence factor among those evaluated in the study was present in $53(98.1 \%)$ of the 54 strains.

Key words: Aeromonas, in vitro susceptibility, antibiotic resistance, virulence factors.

Palabras clave: Aeromonas, susceptibilidad in vitro, resistencia antimicrobiana, factores de virulencia.
Instituto de Medicina Tropical "Pedro Kourí", La Habana, Cuba.

(LB, AF, JL, MR, AA, FAN, YC). Centro Municipal de Higiene y Epidemiología de Güines, La Habana, Cuba (LEC).

Recibido: (2ª versión): 29 de enero de 2010

Aceptado: 10 de febrero de 2011

Correspondencia a:

Laura Bravo

laura@ipk.sld.cu

\section{Introducción}

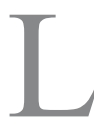

os miembros del género Aeromonas están ubicados taxonómicamente en la familia Aeromonadaceae, son bacilos gramnegativos, anaerobios facultativos, que fermentan y oxidan glucosa, no utilizan lactosa, al menos rápidamente, son oxidasa positiva y están integrados por dieciocho geno-especies o grupos de hibridación ${ }^{1}$.

Son habitantes de ecosistemas acuáticos que incluyen aguas subterráneas, aguas potables y de tratamiento, sistemas de distribución de aguas, plantas de reservas de aguas, así como lagos y ríos contaminados. También se han encontrado en ambientes marinos con bajas concentraciones de sodio, en estuarios, en el suelo, sedimentos, en alimentos incluyendo carne, pescado, vegetales, leche natural y aves comestibles.

Este agente desempeña un papel importante como patógeno primario del tracto gastrointestinal. También es capaz de producir infecciones tales como bacteriemia, septicemia, meningitis, neumonía, peritonitis, miocarditis, síndrome hemolítico urémico (SHU), infecciones hepatobiliares, respiratorias, oculares, vaginales, fascitis necrosante de heridas y nosocomiales, las cuales en ocasiones pueden comprometer la vida del paciente ${ }^{2}$.

En Cuba las bacterias más frecuentemente involucradas en diarreas son especies de Salmonella, Shigella y algunos serotipos de Escherichia coli ${ }^{3}$. La introducción de la prueba de oxidasa para el estudio de los bacilos gramnegativos anaerobios facultativos por parte del
Laboratorio Nacional de Referencia de Enfermedades Diarreicas Agudas del Instituto de Medicina Tropical "Pedro Kourí" (LNR/EDA/IPK) en todos los laboratorios de la Red Nacional de Microbiología, ha permitido agregar a esta lista los microorganismos pertenecientes al género Aeromonas, los cuales han sido reconocidos por la Agencia de Protección del Medio Ambiente de Estados Unidos como patógenos emergentes y la Organización Mundial de la Salud los considera como microorganismos de riesgo $\mathrm{II}^{4,5}$.

Los mecanismos de patogenicidad en las infecciones causadas por especies de este género, tales como colonización, invasión y proliferación, le confieren la habilidad para causar daño en los tejidos del hospedero, así como evadir su sistema inmune. Se ha identificado una serie de factores de virulencia como hemaglutininas y productos extracelulares (toxinas, enzimas y sideróforos, entre otros). A su vez, un gran número de estructuras de la superficie celular parece tener un papel importante en la patogénesis de las infecciones intestinales y sistémicas, haciendo más complejo el cuadro clínico del paciente ${ }^{6}$.

El carácter autolimitado de las infecciones entéricas provocadas por Aeromonas sp reserva la terapia antimicrobiana para los casos de disentería grave, diarrea de duración prolongada, pacientes inmunocomprometidos con enfermedades subyacentes y ante una evidencia de infección extra-intestinal.

Los estudios de susceptibilidad antimicrobiana se realizan, además, con fines investigativos y epidemio- 
lógicos ${ }^{7}$. Desde hace dos o tres décadas se ha reportado un incremento de la resistencia bacteriana, incluso como un fenómeno cambiante, siendo necesaria la vigilancia antimicrobiana sistemática ${ }^{8,9}$. Nuestro país no está ajeno a esta situación, por lo que es de gran utilidad conocer el comportamiento de las especies del género Aeromonas frente a los diferentes agentes antimicrobianos.

Por lo antes expuesto y por la importancia que reviste a nivel nacional e internacional la vigilancia microbiológica y epidemiológica de los microorganismos pertenecientes al género Aeromonas, así como la emergencia y diseminación de cepas resistentes a antimicrobianos, nos propusimos realizar un estudio descriptivo con el objetivo de conocer la susceptibilidad antimicrobiana y los factores de virulencia presentes en cepas aisladas de pacientes con enfermedad diarreica aguda en Cuba.

\section{Materiales y Métodos}

Se estudiaron 54 cepas de Aeromonas spp. referidas al LNR/EDA/IPK desde 14 Centros Provinciales de Higiene, Epidemiología y Microbiología de Cuba y del Municipio Isla de la Juventud, durante los años 2006 y 2007. Las cepas se aislaron a partir de heces de niños bajo cinco años de edad, con un cuadro clínico de diarrea aguda.

Las cepas preservadas en el medio de conservación para enterobacterias "Pasteur", elaborado siguiendo las instrucciones del Manual de Medios y Reactivos del Instituto Pasteur (1978), se inocularon en caldo cerebrocorazón y se incubaron en aerobiosis a $37^{\circ} \mathrm{C}(18-24 \mathrm{~h})$. Posteriormente, se sembró una asada del cultivo en caldo en placas de agar MacConkey y agar sangre de cordero al $5 \%$, incubándose en las mismas condiciones ya descritas anteriormente. Al día siguiente se inocularon por punción ("picadura") y estría en los medios de diferenciación primaria: agar hierro y dos azúcares de Kligler (AHK) y agar hierro lisina (AHL). Ambos se incubaron en aerobiosis a $37^{\circ} \mathrm{C}$, durante 18 a $24 \mathrm{~h}^{10,11}$.

A todas las cepas con perfil bioquímico característico del género se les investigó la presencia de la enzima citocromo oxidasa, según el método de Kovacs, y fueron identificadas hasta el nivel de especie aplicando los esquemas Aeroesquema y Aerokey II $^{12,13}$.

Se determinó la susceptibilidad in vitro a antimicrobianos de las cepas de Aeromonas spp de origen intestinal y extra-intestinal mediante el método de difusión en agar (Kirby-Bauer). Para la lectura e interpretación de los halos de inhibición se utilizaron los protocolos recomendados por el Instituto de Normas de Laboratorio Clínico (CLSI) 2007. Los antimicrobianos estudiados y carga por disco fueron: ácido nalidíxico $30 \mu \mathrm{g}$; amikacina $30 \mu \mathrm{g}$; amoxicilina $25 \mu \mathrm{g}$; ampicilina $10 \mu \mathrm{g}$; aztreonam $30 \mu \mathrm{g}$; carbenicilina $100 \mu \mathrm{g}$; cefalotina $30 \mu \mathrm{g}$; ceftazidima $30 \mu \mathrm{g}$; ceftriaxona $30 \mu \mathrm{g}$; ciprofloxacina $5 \mu \mathrm{g}$; cloranfenicol $30 \mu \mathrm{g}$; doxiciclina
$30 \mu \mathrm{g}$; estreptomicina $10 \mu \mathrm{g}$; gentamicina $10 \mu \mathrm{g}$; kanamicina $30 \mu \mathrm{g}$; penicilina $10 \mu \mathrm{g}$; sulfonamida $250 \mu \mathrm{g}$ y tetraciclina $30 \mu \mathrm{g}$. Multiresistencia fue definida como la resistencia a tres o más clases de antimicrobianos ${ }^{14}$.

Se buscaron los siguientes factores de virulencia: producción de enzimas extracelulares (ADNasa, gelatinasa, elastasa, lecitinasa y hemolisina), titulación de la $\beta$-hemolisina y presencia de fimbrias.

La actividad de ADNasa fue investigada según MaFaddin ${ }^{16}$. Un halo rosado alrededor de las colonias en agar ADNasa con $0,01 \%$ de azul de toluidina indicó actividad de nucleasa. La enzima elastasa se determinó por la metodología establecida por Scharman ${ }^{17}$. Se inoculó por punteo en la superficie del medio de agar elastina, y se incubó hasta siete días a $37^{\circ} \mathrm{C}$. Un halo transparente alrededor de la colonia indicó la presencia de la enzima elastasa. El estudio de la enzima gelatinasa se realizó por la técnica descrita por Blazevic ${ }^{18}$. Se inoculó por punteo en la superficie del medio agar gelatina y se incubó 24 horas a $37^{\circ} \mathrm{C}$. Una opacidad alrededor de la colonia indicó producción de la enzima gelatinasa. La presencia de la enzima lecitinasa se determinó por el protocolo recomendado por Boico ${ }^{19}$. Se inoculó por punteo en la superficie del medio agar lecitina y se incubó 48 horas a $37^{\circ} \mathrm{C}$. Un halo transparente alrededor de la colonia mostró la presencia de esta enzima. Para investigar la presencia de $\beta$-hemolisina, se siguió la técnica descrita por Robinson ${ }^{20}$. Se tomó una asada del cultivo y se inoculó en agar triptona triptona-soya suplementado con sangre de cordero al 5\%. Se incubó 24 horas a $37^{\circ} \mathrm{C}$. Un halo transparente alrededor de la colonia indicó la presencia de esta enzima. Para la titulación de la $\beta$-hemolisina, se siguió la técnica de Burke $^{21}$. Se tomaron $100 \mu \mathrm{L}$ de filtrado libre de células y se realizaron diluciones seriadas en solución salina en placas de microtitulación de 96 pocillos, en forma de U, y se enfrentaron a un volumen igual de una suspensión de eritrocitos de conejo al 1\%. La lectura se realizó en microscopio estereoscópico. Los títulos fueron dados como la última dilución donde había $50 \%$ de hemólisis. Se consideró positiva aquella reacción con un título mayor o igual a 1:4. Para determinar la presencia de fimbrias se procedió según el método de Nishikawa ${ }^{22}$. Se preparó una suspensión bacteriana y una suspensión al 3\% de eritrocitos humanos grupo $\mathrm{O}$ de varios donantes. Se mezcló $20 \mu \mathrm{L}$ de ambas en una lámina, se agitó durante 5 minutos y se consideraron positivas aquellas pruebas en las que se observó aglutinación de los eritrocitos.

Análisis estadístico: Se realizaron usando pruebas de proporciones para comparar los porcentajes. Para ello fue empleado el paquete de programas EPIINFO, versión $6.04^{15}$. En todos los casos las diferencias se consideraron estadísticamente significativas cuando se obtuvo un valor de $\mathrm{p}<0,05$. 


\section{Resultados}

Las 54 cepas estudiadas mostraron el perfil bioquímico característico, quedando ubicadas en el género Aeromonas familia Aeromonadaceae. Se identificaron las especies Aeromonas caviae 23 (42,6\%), Aeromonas hydrophila 10 (18,5\%), Aeromonas veronii biovar sobria 2 (3,7\%), Aeromonas veronii biovar veronii $1(1,9 \%)$ y Aeromonas spp. $18(33,3 \%)$

Al analizar la susceptibilidad in vitro a antimicrobianos de las 54 cepas de Aeromonas analizadas (Tabla 1), encontramos un predominio de cepas resistentes a penicilina, amoxicilina, ampicilina, carbenicilina y cefalotina $(\mathrm{p}<$ $0,01)$. Sin embargo, no se encontró diferencia significativa entre el porcentaje de cepas resistentes y sensibles a tetraciclina y sulfonamida $(\mathrm{p}>0,05)$.

Para el resto de los fármacos predominaron las cepas sensibles sobre las resistentes $(p<0,01)$. Es de destacar que entre estos últimos, amikacina, aztreonam, ceftazidima, ceftriaxona, ciprofloxacina y gentamicina fueron los antimicrobianos que mostraron los mayores niveles de sensibilidad, al ser la frecuencia de cepas sensibles en todos mayor a $90 \%$.

$\mathrm{Al}$ comparar las frecuencias de los aislados resistentes entre Aeromonas caviae, A. hydrophila, y Aeromonas spp., para cada fármaco, encontramos diferencias significativas sólo para sulfonamida $(\mathrm{p}<0,05)$ siendo la frecuencia de aislados resistentes menor para $A$. caviae, y similar entre A. hydrophila, y Aeromonas sp $(\mathrm{p}>0,05)$. Es necesario subrayar que en penicilina y ampicilina se encontraron los porcentajes mayores de resistencia para todas las especies mientras que los más bajos para todas especies se encontraron con ciprofloxacina, ceftriaxona, ceftazidima y aztreonam (Tabla $2)$. Del total de cepas estudiadas $(n=54) ; 26(48,1 \%)$ resultaron multi-resistentes. Se obtuvieron 7 patrones de multi-resistencia en A. caviae, 6 en Aeromonas spp y 6 en $A$. hydrophila. Las especies $A$. veronii biovar sobria y $A$. veronii biovar veronii no presentaron multiresistencia (Tabla 3).

Tabla 1. Susceptibilidad in vitro de 54 cepas de Aeromonas spp a antimicrobianos. Cuba 2006-2007

\begin{tabular}{|c|c|c|c|c|c|c|}
\hline \multirow[t]{3}{*}{ Antimicrobiano } & \multicolumn{6}{|c|}{ Patrón de susceptibilidad in vitro } \\
\hline & \multicolumn{2}{|c|}{ Sensible } & \multicolumn{2}{|c|}{ Intermedio } & \multicolumn{2}{|c|}{ Resistente } \\
\hline & $\mathbf{n}$ & $\%$ & $\mathbf{n}$ & $\%$ & $\mathbf{n}$ & $\%$ \\
\hline CAZ & 52 & 96,3 & 1 & 1,8 & 1 & $1,8^{*}$ \\
\hline CRO & 52 & 96,3 & 1 & 1,8 & 1 & $1,8^{*}$ \\
\hline AK & 50 & 92,6 & 1 & 1,8 & 3 & $5,5^{*}$ \\
\hline ATM & 50 & 92,6 & 3 & 5,5 & 1 & $1,8^{*}$ \\
\hline CIP & 50 & 92,6 & 4 & 7,4 & 0 & $0^{*}$ \\
\hline G & 49 & 90,7 & 3 & 5,5 & 2 & $3,7^{*}$ \\
\hline K & 47 & 87,0 & 3 & 5,5 & 4 & $7,4^{*}$ \\
\hline C & 44 & 81,5 & 4 & 7,4 & 6 & $11,1^{*}$ \\
\hline AN & 42 & 77,7 & 0 & 0 & 12 & $22,2^{*}$ \\
\hline DO & 37 & 68,5 & 9 & 16,7 & 8 & $14,8^{*}$ \\
\hline Su & 29 & 53,7 & 6 & 11,1 & 19 & $35,2^{* *}$ \\
\hline$S$ & 28 & 51,8 & 15 & 27,8 & 11 & 20,4 * \\
\hline TET & 28 & 51,8 & 8 & 14,8 & 18 & $33,3^{* *}$ \\
\hline $\mathrm{KF}$ & 10 & 18,5 & 2 & 3,7 & 42 & $77,7^{*}$ \\
\hline CAR & 9 & 16,7 & 2 & 3,7 & 43 & $79,6^{*}$ \\
\hline AMX & 2 & 3,7 & 0 & 0 & 52 & $96,3^{*}$ \\
\hline AMP & 0 & 0 & 1 & 1,8 & 53 & $98,1^{*}$ \\
\hline P & 0 & 0 & 0 & 0 & 54 & $100^{*}$ \\
\hline \multicolumn{7}{|c|}{$\begin{array}{l}\text { Prueba de comparación de proporciones entre las frecuencias de los aislados sensibles y los que se encontraron resistentes a Aeromonas para } \\
\text { cada fármaco. * } \mathrm{P}<0,01{ }^{* *} \mathrm{P} \geq 0,05 \text {. } \\
\text { Ácido nalidíxico (AN); amikacina (AK); ampicilina (AMP); amoxicilina (AMX)) aztreonam (ATM); carbenicilina (CAR); cefalotina (KF); ceftazidima } \\
\text { (CAZ); ceftriaxona (CRO); ciprofloxacina (CIP); cloranfenicol (C); doxiciclina (DO); estreptomicina (S); gentamicina (G); kanamicina (K); penicilina (P) } \\
\text { sulfonamida (Su); tetraciclina (TET). }\end{array}$} \\
\hline
\end{tabular}


Tabla 2. Frecuencia de resistencia in vitro en 54 cepas de Aeromonas spp según especie. Cuba 2006-2007

\begin{tabular}{|c|c|c|c|c|c|c|c|c|c|c|}
\hline \multirow[t]{2}{*}{$\begin{array}{l}\text { Antimicro- } \\
\text { biano }\end{array}$} & \multicolumn{2}{|c|}{$\begin{array}{l}\text { Aeromonas caviae } \\
\qquad(n=23)\end{array}$} & \multicolumn{2}{|c|}{$\begin{array}{l}\text { Aeromonas sp } \\
\quad(n=18)\end{array}$} & \multicolumn{2}{|c|}{$\begin{array}{l}\text { Aeromonas hydrophila } \\
\qquad(\mathrm{n}=10)\end{array}$} & \multicolumn{2}{|c|}{$\begin{array}{c}\text { Aeromonas } \\
\text { veronii bv sobria } \\
(n=2)\end{array}$} & \multicolumn{2}{|c|}{$\begin{array}{c}\text { Aeromonas } \\
\text { veronii bv veronit } \\
(n=1)\end{array}$} \\
\hline & $n$ & $\%$ & $n$ & $\%$ & $n$ & $\%$ & $\mathbf{n}$ & $\%$ & $n$ & $\%$ \\
\hline SM & 6 & 26,1 & 1 & 5,6 & 3 & 30 & 1 & 50 & 0 & 0 \\
\hline AK & 0 & 0 & 3 & 16,7 & 0 & 0 & 0 & 0 & 0 & 0 \\
\hline$K$ & 0 & 0 & 2 & 11,1 & 2 & 20 & 0 & 0 & 0 & 0 \\
\hline G & 0 & 0 & 1 & 5,6 & 1 & 10 & 0 & 0 & 0 & 0 \\
\hline$P$ & 23 & 100 & 18 & 100 & 10 & 100 & 1 & 50 & 1 & 100 \\
\hline AMX & 21 & 91,3 & 17 & 94,4 & 10 & 100 & 1 & 50 & 1 & 100 \\
\hline AMP & 23 & 100 & 18 & 100 & 10 & 100 & 1 & 50 & 1 & 100 \\
\hline CAR & 17 & 73,9 & 13 & 72,2 & 10 & 100 & 2 & 100 & 1 & 100 \\
\hline ATM & 1 & 4,4 & 0 & 0 & 0 & 0 & 0 & 0 & 0 & 0 \\
\hline KF & 18 & 78,2 & 12 & 66,7 & 10 & 100 & 1 & 50 & 0 & 0 \\
\hline CAZ & 0 & 0 & 1 & 5,5 & 0 & 0 & 0 & 0 & 0 & 0 \\
\hline CRO & 0 & 0 & 1 & 5,5 & 0 & 0 & 0 & 0 & 0 & 0 \\
\hline DO & 6 & 26,1 & 2 & 11,1 & 0 & 0 & 0 & 0 & 0 & 0 \\
\hline TET & 7 & 30,4 & 7 & 38,9 & 4 & 40 & 0 & 0 & 0 & 0 \\
\hline Su & 10 & 4,3 & 6 & 33,3 & 3 & 30 & 0 & 0 & 0 & 0 \\
\hline C & 3 & 13 & 3 & 16,7 & 0 & 0 & 0 & 0 & 0 & 0 \\
\hline $\mathrm{CIP}$ & 0 & 0 & 0 & 0 & 0 & 0 & 0 & 0 & 0 & 0 \\
\hline AN & 4 & 17,4 & 5 & 27,8 & 2 & 20 & 0 & 0 & 0 & 0 \\
\hline
\end{tabular}

Tabla 3 Patrones de multi-resistencia encontrados en cepas de Aeromonas spp aisladas en Cuba. Años 2006-2007

\begin{tabular}{llc|} 
Especies & \multicolumn{1}{c}{ Patrones de multi-resistencia } & Frecuencia/Porcentaje \\
Aeromonas caviae & $\mathrm{AMP} / \mathrm{TET} / \mathrm{Su} / \mathrm{KF}$ * & $3(13,0 \%)$ \\
$\mathrm{n}=23$ & $\mathrm{SM} / \mathrm{AMP} / \mathrm{Su} / \mathrm{C} / \mathrm{AN} / \mathrm{KF}$ & $2(8,6 \%)$ \\
& $\mathrm{SM} / \mathrm{AMP} / / \mathrm{TET} / \mathrm{Su} / \mathrm{KF}$ & $2(8,6 \%)$ \\
& $\mathrm{SM} / \mathrm{AMP} / \mathrm{TET} / \mathrm{Su} / \mathrm{C} / \mathrm{AN} / \mathrm{KF}$ & $1(4,3 \%)$ \\
& $\mathrm{AMP} / \mathrm{TET} / \mathrm{Su}$ & $1(4,3 \%)$ \\
& $\mathrm{SM} / \mathrm{AMP} / \mathrm{ATM} / \mathrm{DO} / \mathrm{Su}$ & $1(4,3 \%)$ \\
& $\mathrm{AMP} / \mathrm{AN} / \mathrm{KF}$ & $1(4,3 \%)$ \\
Aeromonas spp & $\mathrm{AMP} / \mathrm{Su} / \mathrm{KF}$ * & $2(11,1 \%)$ \\
$\mathrm{n}=18$ & $\mathrm{AMP} / \mathrm{TET} / \mathrm{AN} / \mathrm{KF}$ & $2(11,1 \%)$ \\
& $\mathrm{K} / \mathrm{AMP} / \mathrm{Su} / \mathrm{C} / \mathrm{AN} / \mathrm{CAZ}$ & $1(5,5 \%)$ \\
& $\mathrm{AMP} / \mathrm{TET} / \mathrm{KF}$ & $1(5,5 \%)$ \\
& $\mathrm{CN} / \mathrm{AMP} / \mathrm{TET} / \mathrm{Su} / \mathrm{C} / \mathrm{KF}$ & $1(5,5 \%)$ \\
Aeromonas hydrophila & $\mathrm{AK} / \mathrm{AMP} / \mathrm{AN}$ & $1(5,5 \%)$ \\
$\mathrm{n}=10$ & $\mathrm{AMP} / \mathrm{Su} / \mathrm{KF}$ & $1(10 \%)$ \\
& $\mathrm{AMP} / \mathrm{TET} / \mathrm{KF}$ & $1(10 \%)$ \\
& $\mathrm{K} / \mathrm{AMP} / \mathrm{TET} / \mathrm{Su} / \mathrm{KF}$ & $1(10 \%)$ \\
& $\mathrm{SM} / \mathrm{AMP} / \mathrm{TET} / \mathrm{KF}$ & $1(10 \%)$ \\
& $\mathrm{AMP} / \mathrm{AN} / \mathrm{KF}$ & $1(10 \%)$ \\
& $\mathrm{K} / \mathrm{AMP} / \mathrm{TE} \mathrm{T} / \mathrm{Su} / \mathrm{AN} / \mathrm{KF}$ & $1(10 \%)$ \\
\hline *Patrón más frecuente & & \\
\hline
\end{tabular}

De las cepas estudiadas 53/54 (98,1\%) presentaron al menos un factor de virulencia, $49(90,7 \%)$ al menos dos y $37(68,5 \%)$ presentaron tres o más. La enzima ADNasa estuvo presente en 48 cepas $(88,9 \%)$, lecitinasa en $45(83,3 \%)$, gelatinasa en $33(61,1 \%)$ y elastasa en 14 $(25,9 \%)$. Trece cepas $(24,1 \%)$ presentaron $\beta$-hemolisina cuyos títulos oscilaron entre 1:8 y 1:256; 17 cepas $(31,5 \%)$ aglutinaron con los eritrocitos humanos y resultaron ser manosa sensibles.

\section{Discusión}

Las especies del género Aeromonas pueden ser aisladas de muestras clínicas, de animales y diferentes ambientes acuáticos. La prevalencia de las diferentes especies de Aeromonas varía según el área geográfica y el método utilizado para su identificación ${ }^{23}$.

La baja incidencia del aislamiento e identificación de Aeromonas en diferentes áreas geográficas pudieran estar influenciadas, entre otros factores, por un inadecuado diagnóstico microbiológico ${ }^{24,25}$.

Empleando el esquema Aerokey II para la identifi- 
cación de microorganismos pertenecientes al género Aeromonas, en la India, Sinha y cols, caracterizaron siete especies como patógeno único ${ }^{26}$. Los resultados de la presente investigación están acorde a los obtenidos por estos autores, quienes identificaron $A$. caviae, $A$. hydrophila y $A$. veronii biovar sobria a partir de heces de pacientes hospitalizados con diarreas agudas.

En España, Oyvin O y cols, identificaron varias especies del género Aeromonas a partir de 95 muestras de heces, resultando $A$. caviae la especie identificada en mayor porcentaje, resultado que coincide con los del presente estudio ${ }^{27}$.

Desde hace dos o tres décadas se ha reportado un incremento de la resistencia bacteriana, incluso como un fenómeno cambiante, por lo que es necesario la vigilancia antimicrobiana sistemática ${ }^{28,29}$. Nuestro país no está ajeno a esta situación, siendo imprescindible conocer el comportamiento de estas especies frente a los diferentes antimicrobianos.

La terapia antimicrobiana está bien justificada en pacientes inmunocomprometidos, en infecciones extra-intestinales y en aquellos donde la terapia de rehidratación oral no haya sido efectiva. La elevada resistencia a $\beta$ lactámicos (penicilina, amoxicilina, ampicilina, carbenicilina y cefalotina) se corresponde con lo reportado en la literatura científica por diversos autores para los microorganismos de este género ${ }^{31}$. Es importante destacar que la escasa resistencia a quinolonas y cefalosporinas observada puede ser debido a que son antimicrobianos de nueva generación que no han tenido un uso indiscriminado por la población cubana ${ }^{32}$.

En relación a los porcentajes de resistencia obtenidos con sulfonamida, nuestros resultados difieren con los publicados por Herrera ML y cols, en Costa Rica, quienes determinaron la susceptibilidad de cepas del género Aeromonas aisladas de heces de niños ingresados en el Hospital Nacional, arrojando una sensibilidad a sulfonamida de $89 \%{ }^{33}$.

Resultados semejantes a los nuestros obtienen Obi CL y cols, en Sudáfrica en 104 cepas del género Aeromonas aisladas de 309 muestras de origen intestinal, las cuales presentaron una elevada resistencia a penicilina (100\%), amoxicilina (79\%) y ampicilina $(79 \%)^{34}$.

Las cepas de Aeromonas investigadas en este estudio mostraron un elevado porcentaje de resistencia a ampicilina, comportamiento similar al descrito en Tailandia y Filipinas, donde en una investigación llevada a cabo en el año $2005,90 \%$ de las cepas aisladas de diversas fuentes resultaron resistentes a este antimicrobiano ${ }^{35}$.

En relación a los bajos porcentajes de resistencia para ciprofloxacina, ceftriaxona, ceftazidima y aztreonam, los resultados obtenidos en esta investigación coinciden con los obtenidos por Vila J y cols, en Italia en el año 2003, Pokhrel MB y cols, en el 2004 en Nepal y con los estu- dios más recientes publicados en Turquía por Emekdas G y cols, en el 2006 en cepas de Aeromonas aisladas de pacientes con enfermedad diarreica aguda ${ }^{36-38}$.

El nicho ecológico y la frecuencia de uso de antimicrobianos en una región determinada pueden afectar significativamente el perfil de susceptibilidad de las diferentes especies de Aeromonas de diversas áreas geográficas ${ }^{39}$.

En el género en estudio se ha identificado un gran número de estructuras y enzimas extracelulares que tienen un papel importante en la patogenicidad de las infecciones intestinales. Se han realizado numerosos estudios in vitro e in vivo para identificar y caracterizar estos determinantes patogénicos. Muchas son las evidencias que correlacionan la producción de estos factores con el incremento de la patogenicidad ${ }^{40}$.

La $\beta$ hemolisina es considerada uno de los principales factores de virulencia asociados al cuadro diarreico ${ }^{41}$. Un bajo porcentaje de nuestros aislados poseían codificación genética para esta enzima; similares resultados obtuvieron Martins LM y cols, en Brasil al analizar 28 cepas del género Aeromonas, de las cuales $24,1 \%$ la presentó ${ }^{42}$. En cambio Bravo L y cols, obtuvieron $81 \%$ de actividad hemolítica en 27 cepas del género Aeromonas aisladas de 300 niños bajo 5 años de edad con enfermedad diarreica aguda en nuestro país, resultados que difieren de los obtenidos en el presente trabajo ${ }^{43}$

Longa A y cols, en Venezuela determinaron la presencia de $\beta$-hemolisina en $38 \%$ de las 44 cepas aisladas de 397 niños con diarreas, coincidiendo con los resultados obtenidos en este estudio ${ }^{41}$.

Las enzimas extracelulares constituyen importantes factores de virulencia relacionados con la enteropatogenicidad de estas bacterias ${ }^{40}$. La enzima extracelular identificada en mayor proporción en nuestro estudio resultó la ADNasa, seguida de la lecitinasa, gelatinasa y elastasa. Investigaciones realizadas por Abbott SL y cols, sobre la determinación de dichos factores de virulencia en el género Aeromonas evidenciaron la presencia de estas enzimas en los mismos porcentajes ${ }^{44}$.

La técnica de la hemaglutinación reviste gran importancia en este tipo de investigaciones ya que permite detectar la presencia de fimbrias que intervienen en la adherencia de estas bacterias al enterocito ${ }^{45}$. Sólo $31 \%$ de nuestras cepas presentaron hemaglutininas, de las cuales $100 \%$ fue sensible a manosa. Similares resultados publicaron Longa A y cols, al obtener $40 \%$ de cepas capaces de aglutinar con los eritrocitos humanos grupo $\mathrm{O}^{41}$.

En este trabajo se describen algunas propiedades de virulencia de las especies de Aeromonas aisladas de pacientes con enfermedad diarreica en Cuba. Futuras investigaciones deberán relacionarse con los mecanismos genéticos de virulencia y de resistencia en estos microorganismos. 


\section{Resumen}

Se identificaron 54 cepas de Aeromonas aisladas de pacientes con enfermedad diarreica aguda mediante los métodos Aerokey II y Aeroesquema. Se determinó la susceptibilidad antimicrobiana y algunos factores de virulencia. La especie encontrada en mayor frecuencia fue Aeromonas caviae. Se observaron valores de resistencia por sobre $75 \%$ para penicilinas y cefalotina; para el resto de los antimicrobianos estos valores se encontraron bajo $20 \%$; 26 cepas $(48,1 \%)$ presentaron multi-resistencia. Al menos un factor de virulencia de los investigados estuvo presente en $53(98,1 \%)$ de las 54 cepas analizadas.

\section{Referencias}

1.- Morandi A, Zhaxybayeva O, Gogarten P, Graf J. Evolutionary and diagnostic implications of intragenomic heterogeneity in the 16S rRNA gene in Aeromonas strains. J Bacteriol 2005; 187: 6561-4.

2.- Alavandi S V, Ananthan S. Biochemical characteristics, serogrups and virulence factors of Aeromonas species isolated from cases of diarrhoea and domestic water samples in Chenna. Indian J Med Microbiol 2003; 21 (4): 233-8.

3.- Ortiz Bultó P L, Pérez A, Rivero A, Díaz M. Pronóstico bioclimático mensual: enero 2008. Boletín epidemiológico del IPK. 2007; 17 (52) [citado el 31/08/2008]. Disponible en: URL: http://www.ipk.sld.cu/bolepid3/bol52-07.htm

4.- Galindo C L, Chopra A K. Aeromonas and Plesiomonas species. En: Doyle MP, Beuch LR, editores. Food Microbiology: Fundamentals and Frontiers. Washington, DC. ASM Press; 2007: p. 1-56.

5-. Material safety data sheet: infectious substances. Section 1. Infectious agent. Office of laboratory safety, PPHB. 2001 [citado de 20 abril 2004]; Disponible en: URL: http://www.hc-sc.gc.ca/ pphb-dgspsp/msds-ftss/msds6e.html

6.- Arteaga-Garibay R I, Aguilera M G, Navarro A, Molina J, Cravioto A, Valdespino A. El papel del flagelo lateral en la adherencia de Aeromonas spp. Difusión Internacional 2005; 30: 95.

7.- Gutiérrez M, Urbina D, Matiz A, Puello M, Mercado M, Parra M, et al. Comportamiento de la diarrea causada por virus y bacterias en regiones cercanas a la zona ecuatorial. Colombia Médica 2005; 36 (4): 6-14.

8.- Sack R B, Rahaman M, Yumus M. Antimicrobial resistance in organisms causing diarrheal diseases. Clin Infect Dis 1997; 24 (1): 102-5.

9.- Taneja N, Khurana S, Trhan A, Marwaha R K, Sharma M. An outbreak of hospital acquired diarrhea due to Aeromonas sobria. Indian Pediatr 2004; 41: 912-6.

10.- Koneman E W, Allen SD, Dowell V R, Janda WN, Sommers HM, Winn WC. Diagnóstico Microbiológico. $3^{\text {a }}$ ed. Buenos Aires: Editorial Médica Panamericana; 1998.

11.- Martin-Carnahan A, Joseph SW.
Aeromonadaceae. En: Brenner DJ, Krieg NR, Staly JT, Garrity GM, eds. The Proteobacteria, Part B, Bergey's Manual of Systematic Bacteriology. $2^{\text {da }}$. ed. Volumen 2. New York: Springer-Verlag; 2005.p.557-8.

12.- Furuwatari C H, Kawakami Y, Akahame T, Hidaka E, Okimura Y, Nakayama J. Proposal for an aeroscheme (modified Aerokey II) for the identification of clinical Aeromonas species. Med Sci Res 1994; 22: 617-9.

13.- Carnahan A, Behran S, Joseph S. Aerokey II: a flexible key for identifying clinical Aeromonas species. J Clin Microbiol 1991; 29: 2843-9.

14.- Clinical and Laboratory Standards Institute. Performance standards for antimicrobial susceptibility testing: seventeenth informational supplement. Wayne; 2007. CSLI Document M100-S17.Tabla $2^{\mathrm{a}}$ pag: 32-6 and 64.

15.- Dean A G, Dean J A, Coulombier D, Brendel K A, Smith D C, Burton A H, et al. Epi Info Version 6: A Word Processing, Database, and Statistics Program for Epidemiology on Microcomputers. Atlanta, GA: Centers for Disease Control. 1994.

16.- Mc Faddin J F. Pruebas bioquímicas para la identificación de bacterias de importancia clínica. Baltimore: Williams and Wilkins; 2002.

17.- Scharmann W. Elastase in Pseudomonas and Aeromonas Zentralbl Bakteriol 1972; 220 (1): 435-42.

18.- Blazevic D J, Ederer G M. Principles of Biochemical Test in Diagnostic Microbiology. New York, J. Wiley. 1975.

19.- Boiko A V. Pathogenicity factors of various Vibrios and Aeromonas. Zh Mikrobiol Epidemiol Immunobiol 2000; 79 (6): 104-8.

20.- Robinson J. Comparison of direct plating with the use of enrichment culture of isolating of Aeromonas spp. from faeces. J Med Microbiol 1986; 22: 315-7.

21.- Burke J, Robinson J, Atkinson H M, Dibley M, Berry R J, Gracey M. Biochemical characteristics of enterotoxigenic Aeromonas spp. J Clin Microbiol 1982; 15: 48-52.

22.- Nishikawa Y, Kimura T, Kishi T. Mannoseresistant adhesion of motile Aeromonas to INT407 cells and the differences among isolates from humans, food and water. Epidemiol Infect 1991; 107: 171-9.

23.- Bauab T M, Levy C E, Rodríguez J, Pasetto Falcao D. Niche-specific association of
Aeromonas ribotypes from human and environmental origin. Microbiol Immunol 2003; 47 (1): 7-16.

24.- Bravo L, Cabrera L E, Castro G, Ramírez M M, Fernández A, Llop, et al. Aislamiento e identificación de especies pertenecientes a los géneros Aeromonas, Vibrio y Plesiomonas procedentes de muestras extra-intestinales en Cuba. Rev Chil Infect 2007; 24 (3): 204-8.

25.- Bravo L, Valdivia I, Monte R, Ramírez M, García B. Identificación en especie de Aeromonas aisladas en Cuba. Rev Cubana Med Trop 1993; 45 (2): 136-8.

26.- Sinha S, Shimada S, Ramamurthy T, Bhattacharya S K, Yamasaki S, Takeda Y, et al. Prevalence, serotype distribution, antibiotic susceptibility and genetic profiles of mesophilic Aeromonas species isolated from hospitalized diarrhoeal cases in Kolkata, India. J Med Microbiol 2004; 53: 527-34.

27.- Oyvind O, Granum P E, Lassen J, Figueras M J. Lack of agreement between biochemical and genetic identification of Aeromonas spp. APMIS 2005; 113; 203-7.

28.- Sack R B, Rahaman M, Yumus M. Antimicrobial resistance in organisms causing diarrheal diseases. Clin Infect Dis 1997; 24 (1): 102-5.

29.- Taneja N, Khurana S, Trehan A, Marwaha R K, Sharma M. An outbreak acquired diarrhea due to Aeromonas sobria. Indian Pediatrics 2004 41: 912-6 (B).

30.- Gutiérrez J, Nogales MC, Aretio MC, Martin E. The pattern of the sensitivity of Aeromonas spp. that produce extraintestinal infections. An Med Interna 1993; 10 (2): 65-7.

31.- Castro-Escarpulli G, Aguilera Arreola G M, Giono Cerezo S, Hernández-Rodríguez C, Rodríguez Chacon M, Soler Falgás L. El género Aeromonas: ¿Un patógeno importante en México?. Enf Infecc Microbiol 2002; 22 (4): 206-16.

32.- Ramírez M, Valdés N, Bravo L, Fernández A, Castaneda N. Perfil plasmídico y resistencia antimicrobiana en cepas de Shigella aisladas en Cuba. Rev Cub Med Trop 2004; 56 (3): 178-85.

33.- Herrera M L, Vargas A, Moya T, Campos M, Yock I. Aislamientos de Aeromonas hydrophila en el Hospital Nacional de Niños 1995-1998. Rev Méd Hosp Nac Niños (Costa Rica) 2000; 35 (1-2): 73-7. 
34.- Obi C L, Ramalivhana J, Igumbor E O, Samie A. Aeromonas species isolated from clinical samples in the Venda region of South Africa: prevalence, hemolysis, hemagglutination, beta lactamase production and antibiotic susceptibility profiles of the isolates. [ $8^{\text {th }}$ International Symposium on Aeromonas and Plesiomonas. Halifax: 2005].

35.- Petinaki E, Maniati M, Spiliopoulou I, Milona P, Spaliara L. Antimicrobial susceptibility of Aeromonas spp., Vibrio spp and Plesiomonas shigelloides isolates in the Philipines and Thailand. Int J Antimicrob Agents 2005; 25: 345-53.

36.- Vila J, Ruiz J, Gallardo F, Vargas M, Soler L, Figueras MJ, et al. Aeromonas spp and traveler's diarrhea: clinical features and antimicrobial resisitance. Emerg Infect Dis 2003; 9: 552-5.

37.- Pokherl M B, Thapa N. Prevalence of Aeromonas in different clinical and water samples with special reference to gastroenteritis. Nepal Med Coll 2004; 6 (2): 139-43.
38.- Emekdas G, Aslan G, Tezcan S, Serin MS, Yildiz C, Ozturhan H, et al. Detection of the frecuency, antimicrobial susceptibility and genotypic discrimination of Aeromonas strains isolated from municipally treated tap water samples by cultivation and AP-PCR. Int J Food Microbiol 2006 April 1; 107 (3): 310-14.

39.- Janda J M, Abbott S L. Evolving concepts regarding the genus Aeromonas: An expanding panorama of species, disease presentations and unanswered questions. Clin Infect Dis 1998; 27: $332-42$.

40.- Castro-Escarpulli G, Figueras M J, AguileraArreola G, Soler L, Fernández-Rendon E, Aparicio GO, et al. Characterisation of Aeromonas spp isolated from frozen fish intended for human consumption in Mexico. Int Food Microbiol 2003; 4 (1): 41-9.

41.- Longa A, Vizcaya L, Nieves B, Bravo L, Morier L, Pérez I, et al. Factores de virulencia asociados a la enteropatogenicidad en cepas de
Aeromonas spp aisladas de niños con diarrea en Mérida, Venezuela. Rev Cub Med Trop 2005; 57 (2): 85-91.

42.- Martins L M, Marquez R F, Yano T. Incidence of toxic Aeromonas isolated from food and human infection. FEMS Immunol Med Microbiol 2002; 32: 237-42.

43.- Bravo L, San German S, Monte R, Castillo A, Ramírez M, García B. Marcadores fenotípicos en cepas de Aeromonas aisladas en Cuba de niños con enfermedad diarreica aguda. Rev Cub Med Trop 1995, 47 (2): 114-7.

44.- Abbott S L, Ceung W K, Janda J M. The genus Aeromonas: biochemical characteristics, atypical reactions and phenotypic identification schemes. J Clin Microbiol 2003; 41 (6): 2348-57.

45.- Bizani D, Brandelli A. Antimicrobial susceptibility, hemolysis and hemagglutination among Aeromonas spp. isolated from water of a bovine abattoir. Braz J Microbiol 2001; 32 (4): 9-16. 